\title{
New precision era of experiments on strong interaction with strangeness at DAFNE/LNF-INFN
}

T. Ishiwatari ${ }^{1, a}$, M. Bazzi ${ }^{2}$, G. Beer ${ }^{3}$, C. Berucci ${ }^{1,2}$, L. Bombelli ${ }^{4}$, A.M. Bragadireanu ${ }^{1,5}$, M. Cargnelli ${ }^{1}$, C. Curceanu ${ }^{2}$, A. d'Uffizi ${ }^{2}$, C. Fiorini ${ }^{4}$, F. Ghio ${ }^{6}$, C. Guaraldo ${ }^{2}$, R.S. Hayano ${ }^{7}$, M. Iliescu ${ }^{2}$, M. Iwasaki ${ }^{8}$, P. Kienle ${ }^{9}$, P. Levi Sandri ${ }^{2}$, A. Longoni ${ }^{4}$, J. Marton ${ }^{1}$, S. Okada ${ }^{8}$, D. Pietreanu ${ }^{1,5}$, T. Ponta ${ }^{5}$, R. Quaglia ${ }^{4}$, A. Romero Vidal ${ }^{10}$, E. Sbardella ${ }^{2}$, A. Scordo ${ }^{2}$, H. Shi ${ }^{7}$, D.L. Sirghi ${ }^{2,5}$, F. Sirghi ${ }^{2,5}$, H. Tatsuno ${ }^{2}$, A. Tudorache ${ }^{5}$, V. Tudorache ${ }^{5}$, O. Vazquez Doce ${ }^{2,9}$, B. Wünschek ${ }^{1}$, E. Widmann ${ }^{1}$, and J. Zmeskal ${ }^{1}$

\footnotetext{
${ }^{1}$ Stefan-Meyer-Institut für subatomare Physik, Boltzmanngasse 3, 1090 Wien, Austria

${ }^{2}$ INFN, Laboratori Nazionali di Frascati, C.P. 13, Via E. Fermi 40, I-00044 Frascati (Roma), Italy

${ }^{3}$ Department of Physics and Astronomy, University of Victoria, P.O. Box 1700 STN CNC, Victoria BC V8W 2Y2, Canada

${ }^{4}$ Politecnico di Milano, Dipartimento di Elettronica e Informazione, Piazza L. da Vinci 32, I-20133 Milano, Italy

${ }^{5}$ IFIN-HH, Institutul National pentru Fizica si Inginerie Nucleara Horia Hulubei, Reactorului 30, Magurele, Romania

${ }^{6}$ INFN Sezione di Roma I and Instituto Superiore di Sanita, I-00161 Roma, Italy

${ }^{7}$ University of Tokyo, 7-3-1, Hongo, Bunkyo-ku, Tokyo, Japan

${ }^{8}$ RIKEN, Institute of Physical and Chemical Research, 2-1 Hirosawa, Wako, Saitama 351-0198, Japan

${ }^{9}$ Excellence Cluster Universe, Technische Universität München, Boltzmannstraße 2, D-85748 Garching, Germany

${ }^{10}$ Universidade de Santiago de Compostela, Casas Reais 8, 15782 Santiago de Compostela, Spain
}

\begin{abstract}
The strong-interaction shifts and widths of kaonic hydrogen, deuterium, ${ }^{3} \mathrm{He}$, and ${ }^{4} \mathrm{He}$ were measured in the SIDDHARTA experiment. The most precise values of the shift and width of the kaonic hydrogen $1 s$ state were determined to be $\epsilon_{1 s}=-283 \pm$ 36 (stat) \pm 6 (syst) $\mathrm{eV}$ and $\Gamma_{1 s}=541 \pm 89$ (stat) \pm 22 (syst) $\mathrm{eV}$. The upper limit of the kaonic deuterium $\mathrm{K} \alpha$ yield was found to be $\leq 0.39 \%$. In addition, the shifts and widths of the kaonic ${ }^{3} \mathrm{He}$ and ${ }^{4} \mathrm{He} 2 p$ states were determined to be $\epsilon_{2 p}\left({ }^{3} \mathrm{He}\right)=-2 \pm 2$ (stat) \pm 4 (syst) $\mathrm{eV}$ and $\Gamma_{2 p}\left({ }^{3} \mathrm{He}\right)=6 \pm 6($ stat $) \pm 7$ (syst) $\mathrm{eV} ; \epsilon_{2 p}\left({ }^{4} \mathrm{He}\right)=+5 \pm 3$ (stat) \pm 4 (syst) $\mathrm{eV}$ and $\Gamma_{2 p}\left({ }^{4} \mathrm{He}\right)=14 \pm 8$ (stat) \pm 5 (syst) $\mathrm{eV}$. These values are important for the constraints of the low-energy $\bar{K} N$ interaction in theoretical approaches.
\end{abstract}

\section{Introduction}

$\mathrm{X}$-ray spectroscopy of kaonic atoms plays an important role on the fundamental study of the $\bar{K} N$ interaction in the low energy regime. Currently the most interesting topics in this field are measurements of the shifts and widths of the kaonic hydrogen and deuterium $1 s$ states, which are needed for the

\footnotetext{
a e-mail: tomoichi.ishiwatari@ assoc.oeaw.ac.at
} 
extraction of the isospin dependent scattering lengths $\left(a_{0}\right.$ and $\left.a_{1}\right)$. However, these measurements are difficult because of low X-ray yields and large broadening of the peaks.

To overcome this difficulty, new experimental technique was developed by the SIDDHARTA collaboration. Using large-area silicon drift detectors, together with the unique characteristics of the kaon production in the DAФNE $e^{+} e^{-}$collider, X-rays from kaonic hydrogen (and deuterium) were measured with an excellent energy resolution and a good signal-to-background ratio. In addition, we also measured the shifts and widths of the kaonic helium isotope $2 p$ states using gaseous targets for the first time. Detailed descriptions of the experimental methods and the data analysis were reported in [1-5]. Here, we summarize the experimental results obtained in the SIDDHARTA experiment, along with historical overview.

\section{Kaonic hydrogen and deuterium}

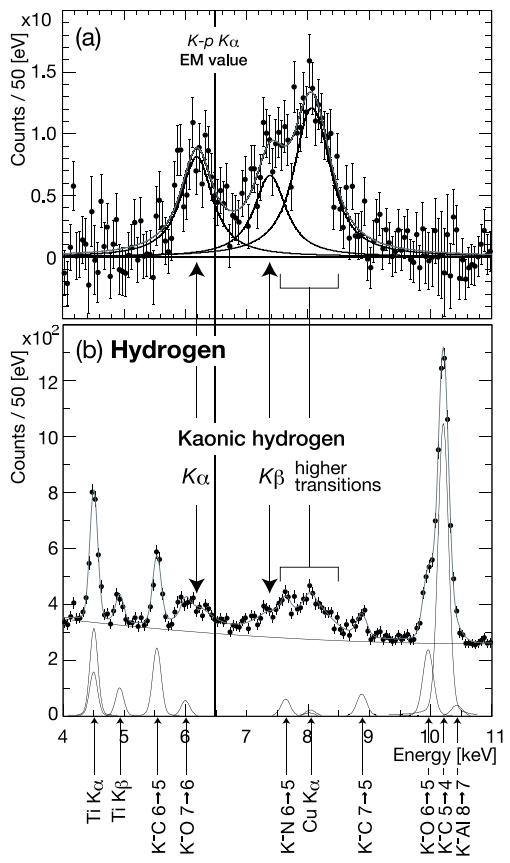

Figure 1. The energy spectra of kaonic hydrogen measured in the SIDDHARTA experiment. (b) shows the energy spectrum without subtraction of the background. The kaonic hydrogen X-ray lines are seen as bumps around the 6-10 keV region. Together with the kaonic hydrogen X-ray signals, several X-ray lines were observed, which were identified as kaonic atom $\mathrm{X}$-rays produced in Kapton. (a) shows the energy spectrum of kaonic hydrogen with subtraction of the continuous background and the kaonic atom $\mathrm{X}$-rays produced in Kapton. The strong-interaction shift and width of the kaonic hydrogen $1 s$ state were determined by the fit of the $\mathrm{K} \alpha$ and $\mathrm{K} \beta$ lines.

There was a serious discrepancy in the sign of the strong-interaction shift of the kaonic hydrogen $1 s$ state. A negative sign is expected in the data analysis of the scattering experiments, while earlier experiments on kaonic hydrogen X-ray spectroscopy gave a positive shift. This discrepancy was known as "the kaonic hydrogen puzzle" [6].

This puzzle was solved by measuring a negative shift in the KpX [6] and DEAR [7] experiments. However, the results of these experiments are not fully consistent. Thus a new precise determination of the shift and width of kaonic hydrogen was awaited to constraint theoretical parameters in the $\bar{K} N$ interaction close to threshold [8].

The SIDDHARTA experiment was performed using large area silicon drift detectors (SDDs) [9] at LNF (Italy). Since the SDDs have a good timing information, beam background can be effectively suppressed together with the detection of $K^{+} K^{-}$pairs in coincidence [1]. Figure 1(b) shows the energy spectrum of kaonic hydrogen measured in the SIDDHARTA experiment. X-ray signals from 
kaonic hydrogen are seen as bumps around the 6-10 keV region. In addition to the kaonic hydrogen $\mathrm{X}$-rays, several peaks were observed, which were produced in the target window material made of Kapton $[1,10]$. Since Kapton contains carbon, nitrogen, oxygen, X-ray transitions from kaonic carbon $\left(K^{-} \mathrm{C}\right)$, nitrogen $\left(K^{-} \mathrm{N}\right)$ and oxygen $\left(K^{-} \mathrm{O}\right)$ were observed [10].

Figure 1(a) shows the energy spectrum of kaonic hydrogen after subtracting the $\mathrm{X}$-ray lines from Kapton and the continuous background. The energy shift and broadening of the kaonic hydrogen peaks were determined by the fit of the energy spectrum. Compared to the QED calculated value, the strong-interaction shift of the kaonic hydrogen $1 s$ state was determined to be

$$
\epsilon_{1 s}=-283 \pm 36(\text { stat }) \pm 6(\text { syst }) \mathrm{eV} \text {. }
$$

The $1 s$ state width was determined to be

$$
\Gamma_{1 s}=541 \pm 89(\text { stat }) \pm 22 \text { (syst) } \mathrm{eV} .
$$

These values are very important for the constraints of the low-energy $\bar{K} N$ interaction. Theoretical calculations using the SIDDHARTA results have been performed, providing new results on the $\bar{K} N$ interaction at the low-energy regions (e.g., $[11,12]$.)

The first measurement of kaonic deuterium was performed [2]. However, due to the limited signalto-background ratio, no clear signals from kaonic deuterium were observed. Using expected values of the shift and width of the kaonic deuterium $1 s$ state, possible X-ray signals from kaonic deuterium were extracted. Compared to Monte Carlo simulations, the upper limit of the kaonic deuterium $\mathrm{K} \alpha$ yield was extracted as

$$
Y(K \alpha)<0.0039 \text { (C.L. 90\%). }
$$

This is consistent with theoretical predictions in cascade models.

\section{Kaonic ${ }^{3} \mathrm{He}$ and ${ }^{4} \mathrm{He}$}

There was a discrepancy in the shift of the kaonic ${ }^{4} \mathrm{He} 2 p$ state [13]. Earlier experiments on kaonic ${ }^{4} \mathrm{He}$ determined a large shift $(-40 \pm 8 \mathrm{eV}$ in average), while an estimated shift derived from kaonic atom data with $Z>2$ is very small $(\sim 0 \mathrm{eV})$. This inconsistency was known as " the kaonic helium puzzle". Related to deeply bound kaonic nuclei, a possible large shift of the order of $10 \mathrm{eV}$ was estimated either in kaonic ${ }^{3} \mathrm{He}$ or ${ }^{4} \mathrm{He} 2 p$ states [14]. Thus, precise measurements both of kaonic ${ }^{3} \mathrm{He}$ and ${ }^{4} \mathrm{He}$ were of interest.

The KEK E570 experiment measured kaonic ${ }^{4} \mathrm{He} \mathrm{X}$-rays with better precision, giving a rather small shift [15]. Although this value is consistent with theoretical estimations, confirmation of this result is needed to clarify the situation.

In the SIDDHARTA experiment, both kaonic ${ }^{3} \mathrm{He}$ and ${ }^{4} \mathrm{He} \mathrm{X}$-rays were measured using a gaseous target for the first time [3-5]. All the previous experiments used a liquid target, requiring a correction of the Compton scattering in helium, whereas the Compton scattering is negligible in the case of the gaseous target. The X-ray peaks of the kaonic ${ }^{3} \mathrm{He}$ and ${ }^{4} \mathrm{He} 3 d \rightarrow 2 p$ transitions were fitted using the Voigt functions. Compared to the QED calculated values, the strong-interaction shifts and widths of the kaonic ${ }^{3} \mathrm{He}$ and ${ }^{4} \mathrm{He} 2 p$ states were determined to be

$$
\begin{array}{lll}
\epsilon_{2 p}\left({ }^{3} \mathrm{He}\right)=-2 \pm 2(\text { stat }) \pm 4(\text { syst }) \mathrm{eV} & \text { and } & \Gamma_{2 p}\left({ }^{3} \mathrm{He}\right)=6 \pm 6(\text { stat }) \pm 7(\text { syst }) \mathrm{eV} \\
\epsilon_{2 p}\left({ }^{4} \mathrm{He}\right)=+5 \pm 3 \text { (stat) } \pm 4 \text { (syst) } \mathrm{eV} & \text { and } & \Gamma_{2 p}\left({ }^{4} \mathrm{He}\right)=14 \pm 8(\text { stat }) \pm 5 \text { (syst) } \mathrm{eV}
\end{array}
$$

These values are in good agreement with the theoretically estimated values, while they are much smaller than the values determined by the earlier experiments [3-5]. No abnormal shift or width was found both in kaonic ${ }^{3} \mathrm{He}$ and ${ }^{4} \mathrm{He}$. Thus, the "kaonic helium puzzle" was solved both in the shift and width, for the first time. 


\section{Summary}

The SIDDHARTA experiment measured the X-rays from light kaonic atoms ranging from hydrogen to ${ }^{4} \mathrm{He}$ using newly developed large area SDDs. Using the time information of the SDDs and the $\mathrm{K}^{+} \mathrm{K}^{-}$ pairs produced by DA $\Phi N E, X$-ray energy spectra of kaonic atoms were obtained with an excellent energy resolution and good signal-to-background ratios. As a result, the most precise values of the shift and width of the kaonic hydrogen $1 s$ state were determined. The first measurement of kaonic deuterium was performed, giving the upper limit of the kaonic deuterium $\mathrm{K} \alpha$ yield. In addition, both the shifts and widths of the kaonic ${ }^{3} \mathrm{He}$ and ${ }^{4} \mathrm{He} 2 p$ states in gaseous targets were determined for the first time. In conclusion, new precision era of experiments on the strong interaction with strangeness at DAFNE/LNF-INFN was successfully created. An upgraded experiment, which is designed for the observation of kaonic deuterium X-rays, is planned in SIDDHARTA-2 at LNF [2].

\section{Acknowledgments}

We thank the DAФNE staff for the excellent working conditions and permanent support. Part of this work was supported by HadronPhysics I3 FP6 Contract No. RII3-CT-2004-506078; HadronPhysics 2, Grant Agreement No. 227431; HadronPhysics 3 Contract No. 283286; Austrian Federal Ministry of Science and Research BMBWK 650962/0001 VI/2/2009; Romanian National Authority for Scientific Research, Contract No. 2-CeX 06-11-11/2006; the Grant-in-Aid for Specially Promoted Research (20002003), MEXT, Japan; DFG Excellence Cluster Universe of the Technische Universität München; and the Austrian Science Fund (FWF): [P20651-N20] and [P24756-N20].

\section{References}

[1] SIDDHARTA Collaboration, Phys. Lett. B 704, 113 (2011)

[2] M. Bazzi et al., Nucl. Phys. A 907, 69 (2013)

[3] SIDDHARTA Collaboration, Phys. Lett. B 697, 199 (2011)

[4] SIDDHARTA Collaboration, Phys. Lett. B 681, 310 (2009)

[5] SIDDHARTA Collaboration, Phys. Lett. B 714, 40 (2012)

[6] M. Iwasaki, et al., Phys. Rev. Lett. 78, 3067 (1997)

[7] G. Beer, et al., Phys. Rev. Lett. 94, 212302 (2005)

[8] W. Weise, Nucl. Phys. A 835, 51 (2010)

[9] M. Bazzi, et al., Nucl. Instrum. Meth. A 628, 264 (2011)

[10] SIDDHARTA Collaboration, et al., arXiv:1306.5912 [nucl-ex], accepted in Nucl. Phys. A, doi:10.1016/j.nuclphysa.2013.07.007

[11] A. Cieply, et al., Phys. Lett. B 702, 402 (2011)

[12] Y. Ikeda, et al., Phys. Lett. B 706, 63 (2011)

[13] C.J. Batty, Nucl. Phys. A 508, 89c (1990)

[14] Y. Akaishi, in: Proc. Inter. Conf. on Exotic Atoms (EXA05), Austrian Academy of Sciences Press, Vienna, 2005, p. 45, doi:10.1553/exa05s45

[15] S. Okada, et al., Phys. Lett. B 653, 387 (2007) 